\title{
The Analysis of the Widom-Rowlinson Model by Stochastic Geometric Methods
}

\author{
J.T. Chayes ${ }^{1}$, L. Chayes ${ }^{1}$, R. Kotecký ${ }^{2}$ \\ 1 Department of Mathematics, UCLA, Los Angeles, CA 90024, USA. Email: jchayes@math.ucla. \\ edu; lchayes@math.ucla.edu \\ 2 Center for Theoretical Study, Charles University, Ovocný trh 3, 11636 Praha 1, Czech Republic \\ E-mail: kotecky@cspuni12.bitnet
}

Received: 7 July 1994

\begin{abstract}
We study the continuum Widom-Rowlinson model of interpenetrating spheres. Using a new geometric representation for this system, we provide a simple percolation-based proof of the phase transition. We also use this representation to formulate the problem, and prove the existence of an interfacial tension between coexisting phases. Finally, we ascribe geometric (i.e. probabilistic) significance to the correlation functions which allows us to prove the existence of a sharp correlation length in the single-phase regime.
\end{abstract}

\section{Introduction}

1A. Background and statement of results. The Widom-Rowlinson model [WR] is a simple and beautiful model of continuum particles. It is of interest both because of it applicability in the description of continuum systems, and because it is the only continuum system for which a phase transition has been rigorously established [R]. The Widom-Rowlinson (WR) model has two equivalent standard formulations - one as a binary gas and the other as a single-species model of a dense (liquid) phase in contact with a rarefied (gas) phase. In the binary gas formulation, the only interaction is a hard-core exclusion between the two species of particles call them $A$ and $B$. There is no intraspecies interaction: two particles of the same type can interpenetrate freely. The phase diagram of the model is a function of the fugacities, $z_{A}$ and $z_{B}$, of the two species. Clearly, there is a symmetry between $A$ and $B$ particles; hence $z_{A}=z_{B}=z$ is a line of symmetry of the phase diagram. For both the continuum and lattice versions of the model, it has been shown via Peierls' arguments that for $z$ large enough, the symmetry is spontaneously broken, yielding two phases: one is $A$-rich and the other is $B$-rich [LG, R]. The transition between these phases is first-order. It is expected, but not proved that the line $z_{A}=z_{B}=z$ of first-order transitions ends in a critical point at some positive value $z=z_{c}$ of the common fugacity. The single-species formulation of the model is obtained by 
integrating out the coordinates of one (say the $B$ ) species (see below). The effective diameter of the particles of the remaining species is then twice the original diameter. The phase transition in the binary formulation maps into a liquid-vapor transition in the single-species version.

The purpose of our work is to show how the geometric ideas can be used to study the phase transition and the interfaces between the coexisting $A$ and $B$ or liquid and gas phases of the Widom-Rowlinson model. The key ingredient in our study is the introduction of a new stochastic representation for the WidomRowlinson measure. We obtain our new representation by first generating percolation configurations of spherical particles, identifying groups of particles with overlapping cores as being in the same cluster. We then "color" each particle either $A$ or $B$. Given the hard-core constraint of the Widom-Rowlinson model, the only configurations which are allowed (i.e. the only ones which receive non-zero weight in the WidomRowlinson measure) are those for which particles in the same cluster are either all $A$ or all $B$. Otherwise, the problem is unconstrained. The result is that all allowed configurations have weights which depend exponentially on the number of clusters within them. Anyone who is familiar with the Fortuin-Kasteleyn representation of the Potts model [FK] should see immediately that our representation does for the Widom-Rowlinson model what the Fortuin-Kasteleyn representation does for the Potts model. Indeed, the symmetries of the Widom-Rowlinson model are manifest in our new representation.

We use our new representation to establish many properties of the WidomRowlinson model. In Sect. 2, we apply percolation methods and use a continutum analog of FKG-domination lemmas to present a new proof of the existence of a phase transition in two and higher dimensions. This proof is completely selfcontained and represents a conceptual simplification of the classic works on the subject [R, LL, GL]. In Sect. 2, we also describe the symmetry-broken phase in terms of percolation and give an appropriate order parameter for the transition. A new proof of the FKG property used for this characterization is given in the Appendix; the original proof can be found in [LM, CGLM]. In Sect. 3, we use monotonicity properties of the representation to establish the existence of an interfacial or surface tension between coexisting phases. This is the first proof of existence of a surface tension in a continuum model. In Sect. 4, we prove the existence of a correlation length for the two-dimensional model in the single-phase regime. In order to do this, we introduce several correlation functions and bound them in terms of each other. Existence of a correlation length is then established using one of these functions.

1B. The model. The Widom-Rowlinson model is a classical statistical mechanics system of interacting particles. To define the model we write the interaction energy, $U_{N}\left(x_{1}, \ldots, x_{N}\right)$, of $N$ particles located at the points $x_{1}, x_{2}, \ldots, x_{N} \in \mathbb{R}^{d}$ as follows: For any $y \in \mathbb{R}^{d}$, we define the halo of $y$ to be the ball of radius $2 a$ with the center at $y, \mathbf{h}(y) \equiv\left\{x \in \mathbb{R}^{d}:|x-y|<2 a\right\}$. The halo of a set $F$ is the union of the halos of its points, $\mathbf{h}(F)=\cup_{y \in F} \mathbf{h}(y)$. The energy $U_{N}\left(x_{1}, \ldots, x_{N}\right)$ of the configuration $\left(x_{1}, \ldots, x_{N}\right)$ is just the difference of the volume $V\left(x_{1}, \ldots, x_{N}\right)$ of the halo $\mathbf{h}\left(x_{1}, \ldots, x_{N}\right)$ and the $\operatorname{sum} \sum_{i=1}^{N}\left|\mathbf{h}\left(x_{i}\right)\right|$,

$$
U_{N}\left(x_{1}, \ldots, x_{N}\right)=V\left(x_{1}, \ldots, x_{N}\right)-N V_{0}
$$

where $V_{0}$ is the volume of a ball of radius $2 a$. Although the interaction (1.1) is (pairwise) attractive, achieving a potential minimum at zero separation, the system 
is, overall, $H$-stable

$$
0 \geqq U_{N}\left(x_{1}, \ldots, x_{N}\right) \geqq-N V_{0},
$$

due to multiparticle effects that saturate the attraction.

The grand-canonical partition function at fugacity $z$ and inverse temperature $\beta$ is defined in the usual fashion: Let $\Lambda \subset \mathbb{R}^{d}$ be the interior of some (regular) finite vessel. We use the notation $\omega_{N} \equiv\left(x_{1}, \ldots, x_{N}\right)$ and $d \omega_{N} \equiv d^{d} x_{1} \cdots d^{d} x_{N}$. Depending on the particular "boundary condition" $\eta$, we write $V^{\eta}\left(\omega_{N}\right)$ (and correspondingly $\left.U_{N}^{\eta}\left(\omega_{N}\right)\right)$. Three natural boundary conditions are: free $(F)$, attractive $(A)$, and repulsive $(R)$. These are introduced by taking $V^{F}\left(\omega_{N}\right)=\left\{\mathbf{h}\left(\omega_{N}\right) \cap \Lambda\right\}$, $V^{R}\left(\omega_{N}\right)=\left|\mathbf{h}\left(\omega_{N}\right)\right|$, and $V^{A}\left(\omega_{N}\right)=\left|\mathbf{h}\left(\omega_{N}\right) \cap \Lambda_{a}\right|$, where $\Lambda_{a} \subset \Lambda$ is obtained by deleting from $\Lambda$ all points within a distance $a$ of the boundary $\partial \Lambda$. It is not hard to see that attractive boundary conditions actually favor the presence of particles near the boundary while repulsive boundary conditions tend to repel them. Then the grand-canonical partition function is

$$
\Xi_{\Lambda}^{(1) \eta}(z, \beta)=\sum_{N} z^{N} Z_{\Lambda, N}^{(1 \eta}(\beta)
$$

where

$$
Z_{\Lambda, N}^{(1)}(\beta)=\frac{1}{N !} \int_{\Lambda} d \omega_{N} e^{-\beta U_{N}^{\eta}\left(\omega_{N}\right)} .
$$

$A$ priori this model does not seem to have any distinguishing features that would make the study of the liquid-gas transition particularly tractable.

In an equivalent formulation of the model (also introduced in [WR]), one considers two species of particles, $A$ and $B$. Here, there is a hard-core exclusion between $A$ and $B$ particles but no interaction between pairs of $A$ or pairs of $B$ particles. Formally, if there are particles located at the positions $x_{1}$ and $x_{2}$, we may put

$$
V_{A A}\left(x_{1}, x_{2}\right)=V_{B B}\left(x_{1}, x_{2}\right)=0
$$

and

$$
V_{A B}\left(x_{1}, x_{2}\right)=\left\{\begin{array}{l}
0 \text { if }\left|x_{1}-x_{2}\right|>2 a \\
\infty \text { otherwise }
\end{array}\right.
$$

The grand canonical partition function, at fugacities $z_{A}$ and $z_{B}$ in a volume $\Lambda$ is given by

$$
\Xi_{\Lambda}^{(2) \eta}\left(z_{A}, z_{B}\right)=\sum_{M, N} z_{A}^{M} z_{B}^{N} Z_{\Lambda, M, N}^{(2 \eta}
$$

where

$$
Z_{\Lambda, M, N}^{\text {(2) } \eta}=\frac{1}{M ! N !} \int_{\Lambda} d \omega_{M}^{A} d \omega_{N}^{B} \chi^{\eta}\left(\omega_{M}^{A}, \omega_{N}^{B}\right)
$$

with $\chi \eta\left(\omega_{M}^{A}, \omega_{N}^{B}\right)$ defined to be zero if the above described hard-core condition is violated in the configuration $\left(\omega_{M}^{A}, \omega_{N}^{B}\right)$ with the boundary condition $\eta$, and unity otherwise. Relevant here are the " $A$-only" boundary conditions, where each point of $\partial \Lambda$ is deemed to be occupied by an $A$ particle, the " $B$-only," and the free boundary conditions.

Equations (1.3) and (1.7) enable one to define the grand-canonical Gibbs measures $\mu_{\Lambda, z, \beta}^{(1) \eta}$ and $\mu_{\Lambda, z_{A}, z_{B}}^{(2) \eta}$. 
Equivalence of the two-component system (1.5) - (1.8) to the one-component model (1.1)-(1.4) is readily demonstrated. Simply fix the configuration $\omega_{M}^{A}$ of $A$ particles and integrate over configurations $\omega_{N}^{B}$ of $B$ particles. It is seen that each of the $B$ particles moves freely through the region $\Lambda \backslash \mathbf{h}\left(\omega_{M}^{A}\right)$, yielding a factor of $\left|\Lambda \backslash \mathbf{h}\left(\omega_{M}^{A}\right)\right|$ to the $N^{\text {th }}$ power. Summing over $N$ gives, up to irrelevant constants, the partition function (1.4) with interaction (1.1) at an effective temperature given by $z_{B}=\beta$. Here, as it turns out, the measure $\mu_{\Lambda, z_{A}, z_{B}}^{(2) \eta}$ transforms, after integrating out the $B$-particles, into the measure $\mu_{\Lambda, z=z_{A} e^{-\beta V_{0}, \beta=z_{B}}}^{(1) \bar{\eta}}$, where $\eta=A$ boundary conditions transform into the attractive boundary conditions, $\bar{\eta}=A, B$ into repulsive, and free into free boundary conditions. In the next section we will define yet a third representation for this model.

\section{The Phase Transition}

2A. The Gray Representation. We call the proposed new description of the Widom Rowlinson system the gray or color-blind representation. Let $\Lambda \subset \mathbb{R}^{d}$ and, for the moment, let us ignore the boundary conditions on $\Lambda$. Also, in order to simplify this section, let us consider only hypercubic ${ }^{1}$ subsets of $\mathbb{R}^{d}$. Denote by $\omega_{N}=\left(x_{1}, \ldots, x_{N}\right)$ any configuration of $N$ points in $\Lambda$ and by $s_{N}$ any of the $2^{N}$ conceivable colorings (i.e. assignments of the $A$ and $B$ labels) of the $N$ given particles. Weighting $A$ and $B$ particles equally, we may write the configurational partition function for the $N$ particles as

$$
Z_{\Lambda, N}^{\circledR \eta}=\frac{1}{N !} \int_{s_{N}, \omega_{N}} d \omega_{N} d s_{N} \chi_{N}^{\eta}\left(\omega_{N}, s_{N}\right)
$$

where

$$
\chi_{N}^{\eta}\left(\omega_{N}, s_{N}\right)=\left\{\begin{array}{l}
1 \text { if the configuration }\left\{\omega_{N}, s_{N}\right\} \text { is "allowed" } \\
0 \text { if the configuration }\left\{\omega_{N}, s_{N}\right\} \text { is "forbidden" }
\end{array}\right.
$$

with given boundary condition $\eta$. For any particle $k=1, \ldots, N$, centered at any point $x_{k} \in \Lambda$, we may define the core region

$$
\mathbf{c}\left(x_{k}\right)=\left\{x \in \mathbb{R}^{d}|| x-x_{k} \mid<a\right\} .
$$

If $\omega_{N}$ is a configuration, the set

$$
\mathbf{c}\left(\omega_{N}\right)=\bigcup_{x_{k} \in \omega_{N}} \mathbf{c}\left(x_{k}\right)
$$

consists of distinct components or clusters. Two particles in $\omega_{N}$ are said to be core connected if they belong to the same component.

It is evident that if $\mathbf{c}\left(x_{i}\right)$ and $\mathbf{c}\left(x_{j}\right)$ overlap for some $i$ and $j$, then $\chi_{N}^{\eta}\left(\omega_{N}, s_{N}\right)$ will vanish unless $i$ and $j$ belong to the same species. This observation obviously generalizes: each separate cluster of $\omega_{N}$ must be composed of a single species. The number of ways in which this can be arranged is clearly $2^{\mathscr{C}^{\eta}\left(\omega_{N}\right)}$, where

$$
\mathscr{C}^{\eta}\left(\omega_{N}\right)=\# \text { of components of } \mathbf{c}\left(\omega_{N}\right) \text { with the boundary condition } \eta \text {. }
$$

\footnotetext{
${ }^{1}$ It turns out, however, that all our results hold in more generality, e.g. in the case in which we take the thermodynamic limit in the sense of van Hove.
} 
Of course, Eq. (2.5) must be carefully interpreted in the presence of specific boundary conditions. In the simplest cases, the interpretation is straightforward in particular, for free (or periodic) boundary conditions, $\mathscr{C}^{F}\left(\omega_{N}\right)$ (or $\mathscr{C}^{P}\left(\omega_{N}\right)$ ) is just the number of clusters. Also of interest in this section are the " $A$-only" boundary conditions, where each point of $\partial \Lambda$ is deemed to be occupied by an $A$ particle. Here the number of clusters $\mathscr{C}^{A}\left(\omega_{N}\right)$ counts all clusters connected to $\partial \Lambda_{a}=\{x \in \Lambda, \operatorname{dist}(x, \partial \Lambda) \leqq a\}$ as a single cluster. For this system, we will denote the associated gray measure by $\mu_{\Lambda, z}^{(A}(\cdot)$.

Hence we can write $(2.1)$ as

$$
Z_{\Lambda, N}^{\circledR \eta}=\frac{1}{N !} \int_{\omega_{N}} d \omega_{N} 2^{\mathscr{C}^{\eta}\left(\omega_{N}\right)} .
$$

In particular, we may express the relative probability of the "gray" configuration, $\omega_{N}$, as

$$
\operatorname{Pr}_{\Lambda, N}^{\circledR} \eta\left(\omega_{N}\right) \propto \frac{1}{N !} 2^{\mathscr{C} \eta\left(\omega_{N}\right)} d \omega_{N}
$$

We may also define a corresponding grand-canonical gray measure at fugacity $z: \mu_{\Lambda, z}^{(} \eta$. From Eq. (2.7) it is seen that if we consider $\mu_{\Lambda, z}^{\circledR \eta}$ conditioned on the $N$-particle state, then the Radon-Nikodým derivative of this conditional measure relative to the Poisson point process at intensity $z$ (also conditioned on $N$ particles) is precisely $2^{\mathscr{C}^{\eta}\left(\omega_{N}\right)}$.

A major advantage of the color-blind formalism is that it allows a comparison between the WR system and an ideal gas. The comparison we have in mind is the continuum analog of FKG-type dominations that are widely used in lattice systems. For continuum problems we proceed as follows. Let $\omega_{N}=\left(x_{1}, \ldots, x_{N}\right)$ and $\omega_{M}=\left(y_{1}, \ldots, y_{M}\right)$ denote $N$ and $M$ particle configurations, respectively. We say that $\omega_{M} \succ \omega_{N}$ if for each $k$ we can find a $j$ such that $x_{k}=y_{j}$. Somewhat less precisely, $\omega_{M} \succ \omega_{N}$ if $\omega_{M} \supset \omega_{N}$.

An event $\mathscr{A}$ is said to be increasing if, for any $\omega \in \mathscr{A}$, it is the case that $\eta \in \mathscr{A}$ for all $\eta \succ \omega$. In other words, the event $\mathscr{A}$ is never destroyed by adding particles to a configuration in which it occurs. If $\mu_{\Lambda}$ and $v_{\Lambda}$ are two (grand-canonical) measures, we say that

$$
\mu_{\Lambda}(-) \underset{\mathrm{F} \widetilde{K} \mathrm{G}}{\gtrless} v_{\Lambda}(-)
$$

if $\mu_{\Lambda}(\mathscr{A}) \geqq v_{\Lambda}(\mathscr{A})$ whenever $\mathscr{A}$ is an increasing event.

The following is a continuum analog of a result that is standard in discrete systems. (It can also be obtained as a special case of Theorem 9.1 of [P] or Lemma 2.1 of $[\mathrm{J}]$.)

Proposition 2.1. Let $\mu_{\Lambda}(\cdot)$ denote a grand-canonical measure for indistinguishable particles on some $\Lambda \subset \mathbb{R}^{d}$ with $N$-particle conditional measures

$$
d \mu_{\Lambda, N}\left(\omega_{N}\right)=\frac{1}{N !} W_{N}\left(\omega_{N}\right) d \omega_{N}
$$

with $W_{N}\left(\omega_{N}\right)$ a.e. continuous, positive and satisfying the usual stability hypothesis (i.e. $W_{N} \leqq e^{b N}$ for some $\left.b<\infty\right)$. For $y \in \Lambda$, regard $\left(\omega_{N}, y\right)$ as an $N+1$ particle 
configuration, and define

$$
\zeta=\inf _{N, \omega_{N}, y} \frac{W_{N+1}\left(\omega_{N}, y\right)}{W_{N}\left(\omega_{N}\right)} .
$$

Then

$$
\mu_{\Lambda}(\cdot) \underset{\mathrm{FKG}}{\geqq} \mu_{\Lambda, \zeta}^{[I]}(\cdot)
$$

where $\mu_{\Lambda, \zeta}^{[I]}(\cdot)$ is the Poisson point process (ideal gas) of intensity $\zeta$.

Remark. The above should seem reasonable in light of the physical interpretation of $\zeta$ as the probability density in the worst case scenario for adding a particle to $\Lambda$.

Proof. This is a straightforward consequence of the following: Let $S_{1}, \ldots, S_{k}$ denote a sequence of random variables, with each $S_{i} \in\{0,1\}$, and define

$$
\zeta_{i}=\min _{S_{1}, \ldots, S_{l-1}, S_{t+1}, \ldots, S_{k}} \operatorname{Prob}_{S}\left(S_{i}=1 \mid S_{1}, \ldots, S_{i-1}, S_{l+1}, \ldots, S_{k}\right)
$$

Then $\operatorname{Prob}_{S}(-)$ dominates, in the sense of FKG, the Bernoulli $\operatorname{Prob}_{R}(-)$ in which the random variables $R_{i}, i=1, \ldots, k$, are assigned $\operatorname{Prob}_{R}\left(R_{i}=1\right)=\zeta_{i}$. This result, which is easily established by induction, has been proved (or assumed) in many places. A general result along these lines is the subject of the first lemma in [Ru].

For the case at hand, we construct a discrete approximation to the continuum process by considering the lattice problem on $\varepsilon \mathbb{Z}^{d} \cap \Lambda$, where $\varepsilon \mathbb{Z}^{d}$ is the hypercubic lattice with spacing $\varepsilon$. We assign $\{0,1\}$-valued random variables $S_{x}, x \in \varepsilon \mathbb{Z}^{d} \cap \Lambda$, to each of the $M(\varepsilon) \approx|\Lambda| \varepsilon^{-d}$ points in $\varepsilon \mathbb{Z}^{d} \cap \Lambda$, with joint probabilities given by

$$
\operatorname{Prob}\left(S_{x_{1}}=\cdots=S_{x_{N}}=1, S_{x}=0 \text { for } x \neq x_{1}, \ldots, x_{N}\right) \propto \varepsilon^{d N} W_{N}\left(x_{1}, \ldots, x_{N}\right) .
$$

We note that the left-hand side of Eq. (2.10) is identified with the $N$ ! equivalent configurations in which a particle is to be found in the $\varepsilon$-vicinity of each of the points $\left(x_{1}, \ldots, x_{N}\right)$. By hypothesis, $\zeta_{i}(\varepsilon) \geqq \zeta \varepsilon^{d}$, so at each stage of the construction, the discrete measures dominate Bernoulli (percolation) measure at density $p=\frac{\zeta \varepsilon^{d}}{1-\zeta \varepsilon^{d}}$. The desired result now follows by continuity of the $\varepsilon \downarrow 0$ limit.

Corollary. The (gray) WR measures $\mu_{\Lambda, z}^{(\mathrm{F}}(-)$ and $\mu_{\Lambda, z}^{(A)}(-)$ dominate, in the sense of $F K G$, the Ideal Gas at a reduced fugacity $\lambda z$, where, $\lambda=\frac{1}{2}$ and $\frac{1}{2^{5}}$ for $d=1$ and $d=2$, respectively, while, for general dimension, $\lambda(d)$ may be estimated.

Proof. We examine the formula

$$
\frac{W_{N+1}\left(\omega_{N}, y\right)}{W_{N}\left(\omega_{N}\right)}=z 2^{\mathscr{C}^{\eta}\left(\omega_{N}, y\right)-\mathscr{C}^{\eta}\left(\omega_{N}\right)}
$$

The quantity in the exponent is the number of clusters lost (or gained) in $\omega_{N}$ by the placement of an additional particle at the point $y$. This is, at most, 1 in $d=1$, 5 in $d=2$, and, in general dimension, we may estimate $\lambda(d) \geqq \frac{1}{2^{\left(3^{d}-1\right)}}$. 
2B. Proof of the Phase Transition. In this and in the next subsection, we will make precise the connection between percolation (in the gray measure) and symmetry breaking in the $A-B$ problem. As is usual in these sorts of arguments, our starting point is to consider some enormous $\Gamma \subset \mathbb{R}^{d}$ with fixed boundary conditions, and then focus attention on some large $\Lambda \subset \Gamma$. We then attempt to make statements about the situation in $\Lambda$ which are uniform in $\Gamma$. Finally, we allow $\Lambda$ to achieve thermodynamic proportions. The definition of "percolation" that turns out to be correct for this system is as follows.

Definition. Let $\Lambda$ and $\Gamma, \Lambda \subset \Gamma$, be hypercubes centered at the origin and let

$N_{\Lambda, \partial \Gamma}=\#$ of particles in $\Lambda$ that are core-connected to the boundary $\partial \Gamma$.

The fraction of percolating sites is defined as

$$
\rho_{\infty}=\lim _{\Lambda \nearrow \mathbb{R}^{d}} \frac{1}{|\Lambda|} \lim _{\Gamma \nearrow \mathbb{R}^{d}} \mu_{\Gamma}^{\Theta A}\left(N_{\Lambda, \partial \Gamma}\right)
$$

where the limits $\Lambda \nearrow \mathbb{R}^{d}$ and $\Gamma \nearrow \mathbb{R}^{d}$ are taken e.g. in such a way as to minimize the left hand side. (The existence of this limit will be established in Subsect. 2C.)

The principle result of this section now amounts to little more than an observation:

Theorem 2.2. In any dimension $d \geqq 2$, for z sufficiently large, symmetry breaking occurs in the WR model.

Proof. Consider the $\Gamma-\Lambda$ setup as described above with $A$ boundary conditions on $\partial \Gamma$. We claim that it is sufficient to establish percolation in the gray model. (In fact, later we will show that this is the necessary and sufficient condition.) Indeed, in any configuration $\omega$, it is clear that those gray particles in $\Lambda$ which are detached from the boundary $\partial \Gamma$ contribute equally to the $A$ and $B$ particle density, while those in the connected component of $\partial \Gamma$ are forced to be of the $A$-type:

$$
\mu_{\Gamma}^{\text {(2) }}{ }^{A}\left(N_{A, \Lambda}-N_{B, \Lambda}\right)=\mu_{\Gamma}^{\text {(a) }}\left(N_{\Lambda, \partial \Gamma}\right),
$$

where $N_{A, \Lambda} \equiv N_{A, \Lambda}(\omega)$ is the number of $A$ particles inside of $\Lambda$, etc. The stated claim follows immediately from Eqs. (2.12) and (2.13). What remains, then, is to show that, for sufficiently high fugacities, there is percolation in the gray measures. However, by Proposition 2.1, this has been reduced to showing that there is percolation in a non-interacting system.

This well known result is easily demonstrated by dividing $\mathbb{R}^{d}$ up into a hypercubic lattice of cell size $l=\frac{a}{\sqrt{d}}$ and calling a cell "occupied" if it contains a particle. It is seen that when neighboring cells are occupied, their occupants are forced to overlap. It is sufficient, then, to push the occupation probability, $1-\exp \left\{-\lambda z\left|\frac{a}{\sqrt{d}}\right|^{d}\right\}$, past the percolation threshold $p_{c}(d)$ of the $d$-dimensional hypercubic lattice, which is non-trivial once $d \geqq 2$.

2C. Characterization of the Symmetry-Broken Phase via Percolation. The above derivation makes it clear that percolation of cores is the proper characterization of coexistence along the line of symmetry. In this section, we will sharpen this 
notion by first showing that the relevant percolation density has a well-defined limiting value, and then demonstrating that this density is indeed an appropriate order parameter for the transition.

Crucial to our analysis are the FKG properties of the various measures discussed in the Appendix. If $\zeta$ and $\omega$ are particle configurations, the relevant notion for the 1component measure is again that $\zeta>\omega$ if $\zeta \subset \omega$. For the two-component problems, if $\omega \equiv\left(\omega_{A}, \omega_{B}\right)$ and $\zeta \equiv\left(\zeta_{A}, \zeta_{B}\right)$ are particle configurations, we say that $\zeta>\omega$ if $\zeta_{A} \supset \omega_{A}$ and $\zeta_{B} \subset \omega_{B}$. In the Appendix we (re)prove that various conditional finite volume one-component and two-component measures enjoy the FKG property.

Proposition 2.3. Let $\Gamma_{L}$ denote the standard sequence of hypercubes of side $L$ tending to infinity and consider the measures $\mu_{\Gamma}^{(1) A}, \mu_{\Gamma}^{(1) R}, \mu_{\Gamma}^{(2)}$, and $\mu_{\Gamma}^{(2)}$. Then the limiting measures $\mu^{\mathbb{D}^{A}}(-), \mu^{\mathbb{D}^{R}}(-), \mu^{\left({ }^{A} A\right.}(-)$, and $\mu^{(2 B}(-)$ exist. Furthermore, all of these measures are translation and rotation invariant. Finally, the percolation density, defined as in Eq. (2.12), exists.

Proof. We will deal exclusively with the case $\mu_{\Gamma}^{(1)}(-)$; the others follow from similar arguments. Existence of the (vague) limit is straightforward. Indeed, if $L_{1}>L_{2}$, the discussion in the remark following Proposition A.2 gives us that

$$
\mu_{\Gamma_{L_{1}}}^{(1) A}(-) \underset{\mathrm{FKG}}{\lessgtr} \mu_{\Gamma_{L_{2}}}^{(1)}(-),
$$

where, of course, by $\mu_{\Gamma_{L_{2}}}^{(1)}(-)$ we mean its restriction to $\Gamma_{L_{2}}$. Therefore, the existence of limiting expectations for local cylinder events is immediate: it is sufficient to show that if $E_{j}^{K_{j}}$ is the event that there are at least $K_{j}$ particles in the set $S_{J}, j=1, \ldots, n$, (with $S_{j}$ of positive Lebesgue measure) that

$$
\lim _{\Gamma \rightarrow \infty} \mu_{\Gamma}^{(1) A}\left(\bigcap_{j=1}^{n} E_{j}^{K_{j}}\right)
$$

exists. However, this follows by monotonicity.

Translation invariance can be established by the following considerations: Let $\mathscr{A}$ denote an increasing local event and let $\mathscr{A}_{x}$ denote the same event shifted by the vector $x$. We claim that $\mu^{\mathbb{1} A}(\mathscr{A})=\mu^{\mathbb{1} A}\left(\mathscr{A}_{x}\right)$. Indeed, both quantities may be expressed as limits: $\mu^{(1)}\left(\mathscr{A}_{x}\right)=\lim _{L \rightarrow \infty} \mu_{\Gamma_{L}}^{(1)} A_{x}\left(\mathscr{A}_{x}\right)$, etc. Let $\Gamma_{L, x}$ denote the box $\Gamma_{L}$ centered at $x$ and let $L_{1} \subset L_{2} \subset L_{3}$ be chosen in such a way that

$$
\Gamma_{L_{1}} \subset \Gamma_{L_{2}, x} \subset \Gamma_{L_{3}}
$$

Then we have

$$
\mu_{\Gamma_{L_{1}}}^{\text {(1) } A}\left(\mathscr{A}_{x}\right) \geqq \mu_{\Gamma_{L_{2}}, x}^{(1) A}\left(\mathscr{A}_{x}\right) \equiv \mu_{\Gamma_{L_{2}}}^{(1) A}(\mathscr{A}) \geqq \mu_{\Gamma_{L_{3}}}^{(\text {(1) } A}(\mathscr{A}),
$$

which establishes translation invariance.

Rotation invariance follows from a similar argument with the shifted box $\Gamma_{L_{2}, x}$ and event $\mathscr{A}_{x}$ replaced by ones that have been rotated.

Similar considerations apply to the slightly non-local functions

$$
N_{\Lambda, \partial \Gamma}^{A}=\# \text { of } A \text { particles in } \Lambda \text { connected to } \partial \Gamma,
$$


i.e., $\mathscr{N}_{\Lambda, \infty}^{A}=\lim _{\Gamma \rightarrow \infty} \mu_{\Gamma}^{(1) A}\left(N_{\Lambda, \partial \Gamma}^{A}\right)$ exists and depends only on the size (and shape) of $\Lambda$. However, if we divide (a regular) $\Lambda$ into $\Lambda_{1} \cup \Lambda_{2}$ with $\Lambda_{1} \cap \Lambda_{2}=\emptyset$ (or of zero measure), it is clear that $\mu^{(1)} A$ a.s. $\mathscr{N}_{\Lambda, \infty}^{A}=\mathscr{N}_{\Lambda_{1}, \infty}^{A}+\mathscr{N}_{\Lambda_{2}, \infty}^{A}$ from which the existence of the percolation density is immediate.

It is now easy to see that the limit $\rho_{\infty}$ defined in Eq. (2.13) exists in a more general context. Furthermore, from the perspective of the two-component problem, $\rho_{\infty}$ is exactly the excess of the $A$ particle density over the $B$ particle density in the $\mu^{\text {(2) } A}$ measure. With a moment's thought, it is also seen to be the excess of particle density in the $\mu^{\mathbb{1} A}$ measure over the particle density in $\mu^{\mathbb{D} R}$, and therefore may be identified with the density of the condensate. We may thus state

Corollary. For the one-component model along the symmetry line $z=\beta$, and for the two-component model with $z_{A}=z_{B}$, percolation is the necessary and sufficient condition for the symmetry breaking. In particular, if $\rho_{\infty}=0$, the limiting infinitevolume one-component and two-component measures are unique.

Proof. Consider e.g. the one-component case. It is not hard to see that for any collection of events $E_{j}^{K_{j}}, j=1, \ldots, n$, one has

$$
\lim _{\Gamma \rightarrow \infty} \mu_{\Gamma}^{(\mathbb{1} A}\left(\bigcap_{j=1}^{n} E_{j}^{K_{J}}\right)=\lim _{\Gamma \rightarrow \infty} \mu_{\Gamma}^{(1) R}\left(\bigcap_{j=1}^{n} E_{j}^{K_{j}}\right) .
$$

Indeed, the difference in these probabilities as measured in $\mu_{\Gamma}^{(1) A}(-)$ versus $\mu_{\Gamma}^{(\mathbb{D} R}(-)$ can be bounded by the probability, in the gray measure, that at least one of the sets $S_{1}, \ldots, S_{n}$ is connected to $\partial \Gamma$. If $\rho_{\infty}=0$, this tends to zero and thus the two limiting measures coincide. Unicity follows because (the restriction of) any other one-component measure constructed with the same parameters and different boundary conditions lies, in the sense of FKG, between $\mu_{\Gamma}^{(1) A}(-)$ and $\mu_{\Gamma}^{(1) R}(-)$.

\section{Surface Tension}

We now turn our attention to the problem of surface tension in the WidomRowlinson model. Any "definition" of surface tension boils down to an assignment of the free energy (per unit area) necessary to create the interface. Thus we seek a situation where we can compare the free energies of two systems which are identical, save for the enforced presence in one of them of an interface separating two coexisting phases. In cases where the pure phases are related by symmetry (manifest or otherwise), it is usually straightforward to arrange this situation. To this end, we follow the continuum analog of the procedures which, by now, are standard in the study of lattice systems.

To keep things conceptually manageable, we will confine our definitions and explicit derivations to the two-dimensional case.

Definition 3.1. Let $\Lambda_{L, M}$ denote the two-dimensional region

$$
\Lambda_{L, M}=\left\{x \in \mathbb{R}^{2}|| x_{1}|<L,| x_{2} \mid<M\right\}
$$

with the boundary

$$
\partial \Lambda_{L, M}=\left\{x \in \mathbb{R}^{2}|| x_{1}|=L \&| x_{2} \mid<M \text { or }\left|x_{2}\right|=M \&\left|x_{1}\right|<L\right\} .
$$


For the two-component model, we will consider the analog of the Dobrushin boundary conditions by putting an $A$ particle at each point of the upper boundary

$$
\partial \Lambda_{L, M}^{+}=\left\{x \in \partial \Lambda_{L, M} \mid x_{2}>0\right\}
$$

while each point of the lower boundary

$$
\partial \Lambda_{L, M}^{-}=\left\{x \in \partial \Lambda_{L, M} \mid x_{2}<0\right\}
$$

houses a $B$ particle. We use $\Xi_{\Lambda_{M, L}}^{(2) A / B}\left(=\Xi_{\Lambda_{M, L}}^{(2) A / B}(z)\right)$ to denote the partition function at fugacity $z_{A}=z_{B}=z$ for this system.

In the $d$-dimensional case, we let $\mathbf{L}=\left(L_{1}, \ldots, L_{d-1}\right)$ and define $\Xi_{\Lambda_{M, \mathbf{L}}}^{(A / B}$ analogously.

Remark. It is clear that, in the immediate vicinity of the midplane points (i.e. in the two-dimensional case, the points $( \pm L, 0)$ ), these boundary conditions cannot be achieved by the physical presence of $A$ and $B$ particles in $\Lambda_{L, M}^{c}$. These tiny violations of WR physics are of no significant consequence - they simply serve to create the boundary conditions that have the most æsthetic appeal to the authors. In particular, if we use boundary conditions where $A(B)$ particles inhabit the boundary only as low (high) as some $x_{2}=s\left(x_{2}=-s\right), s>a$, respectively, all of the forthcoming derivations remain pretty much intact.

We define the surface tension in two dimensions by

$$
\sigma=-\lim _{L \rightarrow \infty} \frac{1}{L} \lim _{M \rightarrow \infty} \log \left[\frac{\Xi_{\Lambda_{L, M}}^{2 \mathrm{~d} A / B}}{\Xi_{\Lambda_{L, M}}^{(2 A}}\right],
$$

should such a limit exist, and similarly in $d>2$.

Theorem 3.1. The limit in Eq. (3.3) exists. Furthermore, the limit may be taken in the reverse or intermediate order, i.e.

$$
\begin{aligned}
\sigma & =-\lim _{M \rightarrow \infty} \lim _{L \rightarrow \infty} \frac{1}{L} \log \left[\frac{\Xi_{\Lambda_{L, M}}^{\text {(2) }}}{\Xi_{\Lambda_{L, M}}^{(2 A}}\right] \\
& =-\lim _{L \rightarrow \infty} \frac{1}{L} \log \left[\frac{\Xi_{\Lambda_{L, L} A / B}^{(2)}}{\Xi_{\Lambda_{L, L}}^{(2 A}}\right] .
\end{aligned}
$$

Proof. The key observation is that the ratio in Eq. (3.3) has a probabilistic interpretation. Indeed, in any configuration in $\Lambda_{L, M}$ with any boundary condition on $\partial \Lambda_{L, M}$, there are only four types of particles:

(1) particles that are connected (via a core-connected cluster) to $\partial \Lambda_{L, M}^{+}$and $\partial \Lambda_{L, M}^{-}$,

(2) particles that are connected to $\partial \Lambda_{L, M}^{+}$but not to $\partial \Lambda_{L, M}^{-}$, 
(3) particles that are not connected to $\partial \Lambda_{L, M}^{+}$but are connected to $\partial \Lambda_{L, M}^{-}$,

(4) particles that are disconnected from all boundaries.

Clearly, in the two-component $\Xi_{\Lambda_{L, M}}^{(2 A / B}$ ensemble, the type (1) is forbidden.

In order to recover the two-component representation from the color-blind representation of these particle configurations, we identify all particles of type (2) as $A$, all particles of type (3) as $B$, and clusters of particles of type (4) as $A$ or $B$ with probability $\frac{1}{2}$.

Now suppose that, in this gray ensemble, we would choose to identify the particles of type (3) as $A$-particles. A moment's thought shows that this produces the two-component ensemble with $A$-boundary conditions on $\Lambda_{L, M}$ subject to the constraint that no particle is connected to both $\partial \Lambda_{L, M}^{+}$and $\partial \Lambda_{L, M}^{-}$. Since $\Xi_{\Lambda_{L, M}}^{(2) / B}$ is precisely the grand canonical weight of all such configurations, it follows that

$$
\frac{\Xi_{\Lambda_{L, M}}^{(2) A / B}}{\Xi_{\Lambda_{L, M}}^{(2)}}=\mu_{\Lambda_{L, M}}^{\text {(2) } A}\left(\mathscr{K}_{L, M}\right) \text {, }
$$

where $\mathscr{K}_{L, M}$ is the event

$$
\begin{aligned}
& \mathscr{K}_{L, M}=\left\{\omega \mid \text { there is no connection between } \partial \Lambda_{L, M}^{+}\right. \text {and } \\
&\left.\partial \Lambda_{L, M}^{-} \text {by } A \text {-type particles }\right\} .
\end{aligned}
$$

Denoting the expectation on the right-hand side of Eq. (3.4) as $K_{L, M}$, monotonicity in the boundary conditions implies that if $M_{1}>M_{2}$, one has

$$
K_{L, M_{1}} \geqq K_{L, M_{2}} \text {. }
$$

Next, let us place a translate $\Lambda_{L_{1}, M}^{\prime}$ of the volume $\Lambda_{L_{1}, M}$ with $A$ boundary conditions on $\partial \Lambda_{L_{1}, M}^{\prime}$ along side the volume $\Lambda_{L_{2}, M}$ with $A$ boundary conditions on $\partial \Lambda_{L_{2}, M}$ in such a way that their $M$-sided boundaries coincide. This creates an environment that is manifestly dominating, in the sense of FKG, the usual system in $\Lambda_{L_{1}+L_{2}, M}$ with $A$ boundary conditions on $\partial \Lambda_{L_{1}+L_{2}, M}$. However, in the former case, events in the left and right pieces are independent. This is easily demonstrated by showing that the weights for any $\omega \subset \Lambda_{L_{1}, M}^{\prime} \cup \Lambda_{L_{2}, M}$ in the two-component ensemble factor into the corresponding weights for the left and right halves of the configuration.

Putting the above two facts together leads to the inequality

$$
K_{L_{1}+L_{2}, M} \geqq K_{L_{1}, M} K_{L_{2}, M} \text {. }
$$

The desired conclusions now follow from a fairly standard subroutine: Eq. (3.7) implies a subadditive inequality from which it is easy to establish the existence of

$$
\sigma_{M}=\lim _{L \rightarrow \infty}-\frac{1}{L} \log K_{L, M}
$$

along with the a priori bound $K_{L, M} \leqq e^{-\sigma_{M} L}$. The inequality (3.6) implies that

$$
\tilde{\sigma}=\lim _{M \rightarrow \infty} \sigma_{M}
$$

exists. Existence of $\sigma$ and the fact that $\tilde{\sigma}=\sigma$ follows from the monotonicity in $M$ of the $K_{L, M}$ and the a priori bounds. Existence of the surface tension in $d>2$ follows an identical set of arguments, the principal distinctions being notational. 


\section{Correlation Functions}

In this section we introduce various two-point functions for the WR systems. Of course, in any continuous classical system, the two-point function is generally accepted as being the average of the (truncated) pair density operator. Here, partly for convenience and partly for æsthetic appeal, we define the appropriate "density" at $x$ to be unity if the point $x$ is covered by a disk of radius $a$ associated with the corresponding particle. Explicitly, for a configuration $\omega$, we define

$$
\chi_{A}(x ; \omega)=\left\{\begin{array}{l}
1 \text { if } \omega \text { contains an } A \text {-particle within a distance } a \text { of } x, \\
0 \text { otherwise },
\end{array}\right.
$$

and similarly for $\chi_{B}(x ; \omega)$. We use the notation $\chi_{A}(x)=\chi_{A}(x ; \omega)$ in one-component representation as well. In terms of these quantities, we can define (truncated) twoand higher-point functions in the usual fashion.

The one-component, two-component, and gray measures represent continuum stochastic geometric models in their own right. As such, the natural notion of correlation is in terms of connectivity. Thus one might consider the probability, e.g. in the gray measure, that two points lie in the same core-connected cluster. In this section we show that, in the single phase regime, the geometrical and statistical mechanical notions of two-point correlation are intimately related. Explicitly, we show that density-density correlations in the one- and two-component models and density excess-density excess correlations (in the two-component model) are bounded uniformly above and below (and in certain cases equal to) the probability of core connections in the gray measure. Relations of this form have been established previously in the Fortuin-Kasteleyn representation of the Potts model (see e.g. [ACCN]) and the random current representation of the Ising model [A].

In order to define the various correlation functions, let us denote by $\langle-\rangle^{\circledast}$ the expectation with respect to the infinite-volume measure $\mu^{\circledast}, *=1,2, G$.

The statistical mechanical correlation functions we consider are:

i) the density-density correlation functions

$\left\langle\chi_{A}(x) ; \chi_{A}(y)\right\rangle \equiv\left\langle\chi_{A}(x) \chi_{A}(y)\right\rangle-\left\langle\chi_{A}(x)\right\rangle\left\langle\chi_{A}(y)\right\rangle$ and

$\left\langle\chi_{B}(x) ; \chi_{B}(y)\right\rangle$,

where $\langle-\rangle$ may be either the two-component measure or the appropriate $(A$ or $B$ ) one-component measure, since these obviously give the same truncated correlations, and

ii) the density excess-density excess correlation function

$\left\langle\chi_{A}(x)-\chi_{B}(x) ; \chi_{A}(y)-\chi_{B}(y)\right\rangle^{2}$,

while the geometrical correlation functions are:

iii) the core-connected correlation functions

$\mu^{\circledast}\left(T_{x y}\right)$,

where $*$ refers to 1,2 , and $G$, and $T_{x y}$ is the event that $x$ is core-connected to $y$, i.e. that $\mathbf{c}(x)$ and $\mathbf{c}(y)$ are in the same component.

Theorem 4.1. Throughout the single-phase regime, along the line of symmetry, the correlation functions described above are bounded above and below by multiples of one another. Explicitly

1) $\mu^{\circledR}\left(T_{x y}\right)=2 \mu^{(1)}\left(T_{x y}\right)=\mu^{(2)}\left(T_{x y}\right)$,

2) $\left\langle\chi_{A}(x) ; \chi_{A}(y)\right\rangle^{\mathbb{1}}=\left\langle\chi_{A}(x) ; \chi_{A}(y)\right\rangle^{\circledR}$, 
3) $\mu^{\oplus}\left(T_{x y}\right)=\left\langle\chi_{A}(x)-\chi_{B}(x) ; \chi_{A}(y)-\chi_{B}(y)\right\rangle^{\circledR}$,

4) $\frac{1}{2}\left\langle\chi_{A}(0)\right\rangle^{\circledR} \mu^{\circledR}\left(T_{x y}\right) \leqq\left\langle\chi_{A}(x) ; \chi_{A}(y)\right\rangle^{\oplus} \leqq \frac{1}{2} \mu^{\circledR}\left(T_{x y}\right)$.

Proof. 1) By $A B$-symmetry, only half of the configurations which contribute to $\mu^{2}\left(T_{x y}\right)$ also contribute to $\mu^{\mathbb{D}}\left(T_{x y}\right)$ - namely those in which the connection takes place via an $A$-cluster - hence the second equality. That $\mu^{\circledR}\left(T_{x y}\right)=\mu^{\circledR}\left(T_{x y}\right)$ is essentially a tautology - the configurations contributing to $\mu^{(2)}\left(T_{x y}\right)$ are simply colorings of those contributing to $\mu^{\circledR}\left(T_{x y}\right)$.

2) The relation $\left\langle\chi_{A}(x) ; \chi_{A}(y)\right\rangle^{\mathbb{D}}=\left\langle\chi_{A}(x) ; \chi_{A}(y)\right\rangle^{(2)}$ is also essentially tautological, since $\mu^{(1)}$ is just the marginal of $\mu^{(2)}$.

3) First observe that, in the single-phase regime, there is no need to truncate the density excess:

$$
\left\langle\chi_{A}(x)-\chi_{B}(x) ; \chi_{A}(y)-\chi_{B}(y)\right\rangle^{(2)}=\left\langle\left(\chi_{A}(x)-\chi_{B}(x)\right)\left(\chi_{A}(y)-\chi_{B}(y)\right)\right\rangle^{(2)} .
$$

Now, to see that

$$
\mu^{\circledR}\left(T_{x y}\right)=\left\langle\left(\chi_{A}(x)-\chi_{B}(x)\right)\left(\chi_{A}(y)-\chi_{B}(y)\right)\right\rangle^{2},
$$

consider any gray configuration and observe that there are only three possibilities: either $x$ or $y$ is not in a cluster, or $x$ and $y$ are in separate clusters, or $x$ and $y$ are in the same cluster (i.e. $T_{x y}$ occurs). The first case clearly contributes nothing to $\left\langle\chi_{A}(x)-\chi_{B}(x) ; \chi_{A}(y)-\chi_{B}(y)\right\rangle^{2}$. In the second case, we may condition on the "color" of $\mathbf{c}(y)$, and note that we then obtain equal and opposite contributions if c $(x)$ is of "color" $A$ or $B$, resulting in exact cancellation. Finally if $T_{x y}$ occurs, the cluster connecting $x$ and $y$ is either of the $A$-type or the $B$-type, so that both terms of the product $\left(\chi_{A}(x)-\chi_{B}(x)\right)\left(\chi_{A}(y)-\chi_{B}(y)\right)$ are of the same sign.

4) The upper bound is straightforward. Consider the event $T_{x y}$ that $x$ is connected to $y$ in the two-component model - the probability of which is $\mu^{(2)}\left(T_{x y}\right)$. Other than those in $T_{x y}$, the only additional configurations for which $\chi_{A}(x) \chi_{A}(y)$ does not vanish are those for which $x$ and $y$ are in separate $A$-clusters. However, the probability of this (in the two-component measure) is exactly $\frac{1}{4}$ of the probability in the gray measure that $x$ and $y$ are in separate clusters. But this latter quantity is also the probability (again in the two-component measure) of e.g. the event that $x$ is in an $A$-cluster and $y$ is in a $B$-cluster. We thus arrive at

$$
\left\langle\chi_{A}(x) \chi_{A}(y)\right\rangle^{(2)}=\mu^{(2)}\left(T_{x y}\right)+\left\langle\chi_{A}(x) \chi_{B}(y)\right\rangle^{(2)} .
$$

However, by the FKG property of the $\mu^{2}$ measure,

$$
\left\langle\chi_{A}(x) \chi_{B}(y)\right\rangle^{(2)} \leqq\left\langle\chi_{A}(x)\right\rangle^{(2)}\left\langle\chi_{B}(y)\right\rangle^{(2)}
$$

while by symmetry,

$$
\left\langle\chi_{B}(y)\right\rangle^{2}=\left\langle\chi_{A}(y)\right\rangle^{2} \text {. }
$$

Equations (4.4)-(4.6) establish the desired upper bound.

The lower bound will not come so easily. It turns out, however, that in order to prove this bound, it is not necessary to assume translation invariance of the system, provided that the boundary conditions are $A-B$ symmetric. We begin by defining $\chi_{G}(x)$ to be the indicator of the event in the gray representation that $x$ belongs to 
the core of a particle and $\chi_{\emptyset}(x)=1-\chi_{G}(x)$. To simplify the forthcoming algebraic manipulations, it is useful to define the following quantities:

$$
\alpha=\mu^{\oplus}\left(T_{x y}\right)
$$

the probability that $x$ and $y$ are in the same component,

$$
\beta=\left\langle\chi_{G}(x) \chi_{G}(y)\right\rangle^{\circledR}-\mu^{\oplus}\left(T_{x y}\right),
$$

the probability that $x$ and $y$ are in separate components,

$$
\gamma=\left\langle\chi_{G}(x) \chi_{\emptyset}(y)\right\rangle^{\circledR},
$$

the probability that $x$ is in the core region of some particle, but $y$ is not,

$$
\delta=\left\langle\chi_{\emptyset}(x) \chi_{G}(y)\right\rangle^{\circledR},
$$

as above, but with $x$ and $y$ interchanged, and

$$
\varepsilon=\left\langle\chi_{\emptyset}(x) \chi_{\emptyset}(y)\right\rangle^{\oplus},
$$

the probability that neither $x$ nor $y$ are in the core region of any particle.

It is not so hard to see that

$$
4\left[\left\langle\chi_{A}(x) \chi_{A}(y)\right\rangle^{(2)}-\left\langle\chi_{A}(x)\right\rangle^{(2)}\left\langle\chi_{A}(y)\right\rangle^{(2)}\right]=(2 \alpha+\beta)-(\alpha+\beta+\gamma)(\alpha+\beta+\delta) .
$$

After some tedious algebra, the right-hand side can be reexpressed:

$$
\begin{aligned}
& 4\left[\left\langle\chi_{A}(x) \chi_{A}(y)\right\rangle^{2}-\left\langle\chi_{A}(x)\right\rangle^{2}\left\langle\chi_{A}(y)\right\rangle^{(2)}\right] \\
& \quad=\alpha\left(\alpha+\beta+\frac{1}{2}(\gamma+\delta)\right)+\left[\alpha\left(\frac{1}{2}(\gamma+\delta)+2 \varepsilon\right)+\beta \varepsilon-\gamma \delta\right] .
\end{aligned}
$$

We claim that the term in the square brackets above is nonnegative. To see this, we use the following FKG domination, as proved in Proposition A.4 of the Appendix:

$$
\mu^{(2)}\left(-\mid \chi_{A}(x)\right) \underset{\mathrm{FKG}}{\geqq} \mu^{(2)}\left(-\mid \chi_{\emptyset}(x)\right),
$$

where $\chi_{\emptyset}(x)=1-\chi_{A}(x)-\chi_{B}(x)$. As a consequence, we obtain

$$
\left\langle\chi_{A}(y) \mid \chi_{A}(x)\right\rangle^{\circledR} \geqq\left\langle\chi_{A}(y) \mid \chi_{\emptyset}(x)\right\rangle^{\circledR},
$$

i.e.

$$
\frac{\left\langle\chi_{A}(x) \chi_{A}(y)\right\rangle^{2}}{\left\langle\chi_{A}(x)\right\rangle^{2}} \geqq \frac{\left\langle\chi_{A}(y) \chi_{\emptyset}(x)\right\rangle^{2}}{\left\langle\chi_{\emptyset}(x)\right\rangle^{2}}
$$

which translates to

$$
\frac{\frac{1}{2} \alpha+\frac{1}{4} \beta}{\frac{1}{2}(\alpha+\beta+\gamma)} \geqq \frac{\frac{1}{2} \delta}{\delta+\varepsilon}
$$

or

$$
\frac{1}{2} \alpha \delta+\alpha \varepsilon+\frac{1}{2} \beta \varepsilon \geqq \frac{1}{2} \gamma \delta .
$$


Reversing the roles of $x$ and $y$ gives the analogous relation

$$
\frac{1}{2} \alpha \gamma+\alpha \varepsilon+\frac{1}{2} \beta \varepsilon \geqq \frac{1}{2} \gamma \delta
$$

Equations (4.14) and (4.15) together imply the claimed inequality. We thus have

$$
\left\langle\chi_{A}(x) \chi_{A}(y)\right\rangle^{(2)}-\left\langle\chi_{A}(x)\right\rangle^{(2)}\left\langle\chi_{A}(y)\right\rangle^{(2)} \geqq \alpha\left(\alpha+\beta+\frac{1}{2}(\gamma+\delta)\right) \text {. }
$$

Identifying

$$
\begin{aligned}
& \frac{1}{2}(\alpha+\beta+\gamma)=\left\langle\chi_{A}(x)\right\rangle^{2}, \\
& \frac{1}{2}(\alpha+\beta+\delta)=\left\langle\chi_{A}(y)\right\rangle^{(2)}
\end{aligned}
$$

we obtain the desired result

$$
\frac{1}{4}\left[\left\langle\chi_{A}(x)\right\rangle^{(2)}+\left\langle\chi_{A}(y)\right\rangle^{(2)}\right] \mu^{\circledR}\left(T_{x y}\right) \leqq\left\langle\chi_{A}(x) ; \chi_{A}(y)\right\rangle^{(2)}
$$

Corollary. Throughout the single-phase regime, along the line of symmetry, the correlation length $\xi$ defined by

$$
-\frac{1}{\xi}=\lim _{|x| \rightarrow \infty} \frac{1}{|x|} \log \left\langle\chi_{A}(0) ; \chi_{A}(x)\right\rangle^{2}
$$

exists. Here $|x|$ denotes the Euclidean length. Furthermore, the correlation length $\xi$ defined above is equal to the (similarly defined) correlation length for any of the other statistical mechanical or geometrical correlation functions listed in the statement of Theorem 4.1.

Proof. By Theorem 4.1, it is sufficient to establish, for example, the existence of

$$
\lim _{|x| \rightarrow \infty} \frac{1}{|x|} \log \mu^{(2)}\left(T_{0 x}\right) \text {. }
$$

Clearly, for any $y$,

$$
T_{0 x} \supset T_{0 y} \cap T_{y x} .
$$

So, using the FKG inequality (for the two-component measure),

$$
\mu^{(2)}\left(T_{0 x}\right) \geqq \mu^{2}\left(T_{0 y}\right) \mu^{(2)}\left(T_{y x}\right) \text {. }
$$

Hence, for $y \propto x$ with $|y| \leqq|x|$, we can obtain a subadditive inequality, from which the desired result is immediate. 


\section{Appendix. FKG Properties for the Widom-Rowlinson Model}

In this section, we establish FKG properties for the one- and two-component versions of the Widom-Rowlinson model. Most of Proposition A.1 can be found in the references [LM, CGLM], where it was derived in the context of an equivalent spin model; however the present derivation has the appeal that it deals directly with the models at hand, and therefore suggests further results such as those in Proposition A.4.

We begin with the lattice version of the one-component WR model. Let $\Gamma \subset \mathbb{Z}^{d}$ and let $\omega \subset \Gamma$ denote a particle configuration. More formally, for each $i \in \Gamma, \omega(i) \in$ $\{0,1\}$ corresponds to the presence or absence of a particle at the site $i$. If we regard the lattice as being immersed in $\mathbb{R}^{d}$, then (with some attention paid to boundary conditions) we may define $\mathbf{h}(\omega) \equiv \mathbf{h}_{a}(\omega)$ exactly as in the discussion preceding Eq. (1.1). If $\beta, z$, and $a$ are positive real numbers, the finite volume one-component measures WR measures, $\mu_{\Gamma ; a, \beta, z}^{(1)}$, are defined by assigning the weights

$$
\mu_{\Gamma ; a, \beta, z}^{(1)}(\omega) \propto\left(z e^{\beta V_{0}}\right)^{|\omega|} e^{-\beta|\mathbf{h}(\omega)|} .
$$

It is noted that, due to the discreteness of the lattice, the measure defined by Eq. (A.1) is not exactly the measure that one obtains by integrating out the $B$ degrees of freedom in the analogous two-component measure (see Eq. A.6 below).

The following is readily established:

Proposition A.1. For any finite $\Gamma \subset \mathbb{Z}^{d}$ and any positive real numbers $\beta, z$, and $a$, the measures $\mu_{\Gamma ; a, \beta, z}^{(1)}(-)$ are $F K G$ with respect to the natural partial order of $\{0,1\}^{\Gamma}$.

Remark. We refer the readers who are unfamiliar with the basic notations and properties of FKG measures to the expositions in [Gr] or [L]. The original references are $[\mathrm{H}]$ and $[\mathrm{FKG}]$.

Proof. It is sufficient to verify the so-called lattice condition, namely if $\omega_{1} \omega_{2} \subset \Gamma$ and $\left.\omega_{1} \vee \omega_{2}\right|_{i}=\max \left\{\omega_{1}(i), \omega_{2}(i)\right\}$, and $\left.\omega_{1} \wedge \omega_{2}\right|_{i}=\min \left\{\omega_{1}(i), \omega_{2}(i)\right\}$, then

$$
\mu\left(\omega_{1} \vee \omega_{2}\right) \mu\left(\omega_{1} \wedge \omega_{2}\right) \geqq \mu\left(\omega_{1}\right) \mu\left(\omega_{2}\right) .
$$

Thus, given the form of the weights, we must show that

$$
\left|\mathbf{h}\left(\omega_{1} \vee \omega_{2}\right)\right|+\left|\mathbf{h}\left(\omega_{1} \wedge \omega_{2}\right)\right| \leqq\left|\mathbf{h}\left(\omega_{1}\right)\right|+\left|\mathbf{h}\left(\omega_{2}\right)\right|
$$

However, it is clear that

$$
\mathbf{h}\left(\omega_{1} \vee \omega_{2}\right) \subset \mathbf{h}\left(\omega_{1} \cup \omega_{2}\right),
$$

while

$$
\mathbf{h}\left(\omega_{1} \wedge \omega_{2}\right)=\mathbf{h}\left(\omega_{1} \cap \omega_{2}\right) .
$$

Thus the desired inequality is immediate. 
Next we treat the case of the two-component model. Again we restrict to some finite $\Gamma \subset \mathbb{Z}^{d}$, but here we have $\omega \in\{-1,0,+1\}^{\Gamma}$ where, by convention, $\omega(i)=-1$ indicates the presence of a $B$ particle at the site $i, \omega(i)=+1$ an $A$ particle at $i$, while $\omega(i)=0$ means there is no particle at $i$. This notation suggests the partial order

$$
\omega_{1} \succ \omega_{1}^{\prime} \text { if } \forall i \in \Gamma \quad \omega_{1}(i) \geqq \omega_{1}^{\prime}(i) .
$$

For $\omega \in\{-1,0,+1\}^{\Gamma}$, define $\omega^{A}$ and $\omega^{B}$ by the condition $\omega^{B}(i)=1$ if $\omega(i)=-1$ and zero otherwise (i.e. if there is a $B$ particle at the site $i \in \Gamma$ ) and similarly for $\omega^{A}$. As usual, we may also regard these configurations as subsets of $\Gamma$. Again, regarding $\Gamma$ as immersed in $\mathbb{R}^{d}$, we may define $\mathbf{c}\left(\omega^{A}\right)$ and $\mathbf{c}\left(\omega^{B}\right)$ exactly as in Eq. (2.4). For positive real numbers $a, z_{A}$ and $z_{B}$, the two-component WR measures are defined on some finite $\Gamma$ by the weights

$$
\mu_{\Gamma ; a, z_{A}, z_{B}}^{(2}(\omega) \propto z_{A}^{\left|\omega^{A}\right|} z_{B}^{\left|\omega^{B}\right|} \chi\left(\mathbf{c}\left(\omega^{A}\right) \cap \mathbf{c}\left(\omega^{B}\right)=\emptyset\right) .
$$

Proposition A.2. For any finite $\Gamma \subset \mathbb{Z}^{d}$ and any positive, real numbers $a, z_{A}, z_{B}$, the measures $\mu_{\Gamma ; a, z_{A}, z_{B}}^{(-)}(-)$are $F K G$ with respect to the partial order described in Eq. (A.5).

Proof. We will again verify the lattice condition. If $\omega_{1}$ and $\omega_{2}$ are configurations, then $\omega_{1} \wedge \omega_{2}$ consists of those $A$ particles common to both configurations and all of the $B$ particles of either configuration. A similar statement holds for $\omega_{1} \vee \omega_{2}$ with the roles of $A$ and $B$ interchanged. It therefore follows that if $\omega_{1}$ and $\omega_{2}$ are allowed - in the sense that $\mathbf{c}\left(\omega^{A}\right) \cap \mathbf{c}\left(\omega^{B}\right)=\emptyset$ - then so are the configurations $\omega_{1} \vee \omega_{2}$ and $\omega_{1} \wedge \omega_{2}$. Aside from the restriction to allowed configurations, the lattice condition holds as an equality. Thus the desired result is established.

Remark. Since our proofs of the FKG inequalities involve the verification of the lattice condition, the results also follow for measures obtained by conditioning on any cylinder events. In particular, these results also hold for any boundary condition on $\mathbb{Z}^{d} \backslash \Gamma$ that can be constructed with a fixed particle configuration. This of course implies the obvious monotonicity properties for measures on increasing sequences of sets with maximal or minimal boundary conditions.

Passage to the continuum limit is accomplished in a natural fashion. If $\Lambda \subset \mathbb{R}^{d}$, we consider the lattices $\varepsilon \mathbb{Z}^{d}$ and use the sets $\Gamma_{\varepsilon}(\Lambda) \equiv \varepsilon \mathbb{Z}^{d} \cap \Lambda$. The fugacities are rescaled by $z_{A} \rightarrow \varepsilon^{d} z_{A}$, while $a$ remains fixed and $\beta \rightarrow \varepsilon^{d} \beta$. It is not hard to show (e.g. by the arguments of $[\mathrm{CK}]$ ) that the limiting measures which emerge are just the one- and two-component measures introduced in Subsect. 1B. Thus we arrive at:

Corollaries. The one- and two-component WR continuum measures have the FKG property with respect to the continuum analogues of the partial orders described above.

Remark. As in the lattice cases, these corollaries hold for conditional measures that can be obtained by conditioning on any cylinder events.

We now prove the FKG dominance relation that was claimed in Eq. (4.10). Note that this is not a completely trivial result since the indicator $\chi_{\emptyset}(x)$ is not a decreasing function. To prove this relation, we will establish the analogous result on the lattice in finite volume, and then allow the relation to carry over to the 
continuum limit. In order to obtain the relation on the lattice, we invoke a result of [Ho] (a simple proof of which may be found in [L]):

Theorem A.3. Let $\Omega$ denote a finite, partially ordered set and let $\mu$ and $v$ denote probability measures on $\Omega$. Then a sufficient condition for the FKG domination $\mu \underset{\mathrm{FKG}}{\geqq}$ is that

$$
\mu\left(\omega_{1} \vee \omega_{2}\right) v\left(\omega_{1} \wedge \omega_{2}\right) \geqq \mu\left(\omega_{1}\right) v\left(\omega_{2}\right)
$$

holds for all $\omega_{1}, \omega_{2} \in \Omega$.

Using this, we obtain:

Proposition A.4. Let $\Gamma \subset \mathbb{Z}^{d}$ with $|\Gamma|<\infty$ and let $x \in \Gamma$. Define $\chi_{A}(x)$ to be the lattice analogue of the indicator function defined in $E q$. (4.1), and let $\chi_{\emptyset}(x)=$ $1-\chi_{A}(x)-\chi_{B}(x)$. Then, for any fixed boundary condition on $\partial \Gamma$, and for any positive, real numbers $a, z_{A}$ and $z_{B}$, the two-component conditional $W R$ measures, $\mu_{\Gamma ; a, z_{A}, z_{B}}^{(2)}\left(-\mid \chi_{A}(x)\right)$ and $\mu_{\Gamma ; a, z_{A}, z_{B}}^{(2)}\left(-\mid \chi_{\emptyset}(x)\right)$, on $\{-1,0,+1\}^{\Gamma}$ obey the FKG dominance relation:

$$
\mu_{\Gamma ; a, z_{A}, z_{B}}^{(2)}\left(-\mid \chi_{A}(x)\right) \underset{\mathrm{FKG}}{\geqq} \mu_{\Gamma ; a, z_{A}, z_{B}}^{(2)}\left(-\mid \chi_{\emptyset}(x)\right) .
$$

Proof. We will use Theorem A.3 above. To this end, let us write the weights of the configuration $\omega=\left(\omega^{A}, \omega^{B}\right) \in \Omega$ as

$$
\mu_{\Gamma ; a, z_{A}, z_{B}}^{(2)}\left(-\mid \chi_{A}(x)\right) \propto W_{A}(\omega) \equiv z_{A}^{\left|\omega^{A}\right|} z_{B}^{\left|\omega^{B}\right|} \chi\left(\mathbf{c}\left(\omega^{A}\right) \cap \mathbf{c}\left(\omega^{B}\right)=\emptyset\right) \chi_{A}(x ; \omega),
$$

and similarly for $\mu_{\Gamma ; a, z_{A}, z_{B}}^{(2)}\left(-\mid \chi_{\emptyset}(x)\right) \propto W_{\emptyset}(\omega)$. By Theorem A.3, it suffices to establish that for all $\omega_{1}, \omega_{2} \in \Omega$, we have

$$
W_{A}\left(\omega_{1} \vee \omega_{2}\right) W_{\emptyset}\left(\omega_{1} \wedge \omega_{2}\right) \geqq W_{A}\left(\omega_{1}\right) W_{\emptyset}\left(\omega_{2}\right)
$$

since the constants of proportionality appear on both sides of the desired inequality. Similarly, we need not consider the multiplicative factors involving $z_{A}$ and $z_{B}$.

We have already demonstrated the necessary inequalities for $\chi\left(\mathbf{c}\left(\omega^{A}\right) \cap\right.$ $\left.\mathbf{c}\left(\omega^{B}\right)=\emptyset\right)$ in Proposition A.2. Furthermore, because $\chi_{A}(x ; \omega)$ is increasing, it is clear that for any $\omega_{1}, \omega_{2} \in \Omega$, if $\chi_{A}\left(x ; \omega_{1}\right)=1$, then $\chi_{A}\left(x ; \omega_{1} \wedge \omega_{2}\right)=1$. However, a similar statement does not hold for $\chi_{\emptyset}(x, \omega)$ for all $\omega_{1}$ and $\omega_{2}$. However, if $\omega_{1}$ satisfies $\chi_{A}\left(x ; \omega_{1}\right)=1$ and $\chi\left(\mathbf{c}\left(\omega_{1}^{A}\right) \cap \mathbf{c}\left(\omega_{1}^{B}\right)=\emptyset\right)$, the "covering" of $x$ by the $A$-core centered at some $y \in \mathbf{c}(x)$ prevents $\omega_{1}\left(y^{\prime}\right)=-1$ for any other $y^{\prime} \in \mathbf{c}(x)$. Thus, for all $y$ in $\mathbf{c}(x), \omega_{1} \in\{0,+1\}$. Combining this with the constraint $\chi_{\emptyset}\left(x, \omega_{2}\right)=1$, we see that for all $y$ in $\mathbf{c}(x),\left.\omega_{1} \wedge \omega_{2}\right|_{y}=0$. This gives the inequality (A.8), and hence the desired result.

Note added in proof. After completion of this work, we learned about other related work by various groups. In particular, Klein $[\mathrm{K}]$ had already proposed our "gray representation" of generalized continuum percolation in 1982; that a special case of this representation is equivalent to a WR model was noted by Given and Stell [GS]. We are indebted to H.-O. Georgii for pointing out these references to us. Also, in recent independent work, Georgii [Ge] and Giacomin, Lebowitz and Maes [GLM] have used ideas similar to ours to derive results essentially equivalent to those in Sects. $2 \mathrm{~A}$ and $2 \mathrm{~B}$. 


\section{References}

[A] Aizenman, M.: Geometric Analysis of $\Phi^{4}$ Fields and Ising Models I, II. Commun. Math. Phys. 86, 1-48 (1982)

[ACCN] Aizenman, M., Chayes, J.T., Chayes, L., Newman, C.M.: Discontinuity of the Magnetization in One-Dimensional $1 /|x-y|^{2}$ Ising and Potts Models. J. Stat. Phys. 50, 1-40 (1988)

[CGLM] Cassandro, M., Gallavotti, G., Lebowitz, J.L., Monroe, J.L.: Existence and Uniqueness of Equilibrium States for Some Spin and Continuum systems. Commun. Math. Phys. 32, 153-165 (1973)

[CK] Chayes, L., Klein, D.: A Generalization of Poisson Convergence to "Gibbs Convergence" with Applications to Statistical Mechanics. Helv. Phys. Acta 67, 30-42 (1994)

[FK] Fortuin, C.M., Kasteleyn, P.W.: On the Random Cluster Model I. Introduction and Relation to Other Models. Physica 57, 536-564 (1972)

[FKG] Fortuin, C.M., Kasteleyn, P.W., Ginibre, J.: Correlation Inequalities on Some Partially Ordered Sets. Commun. Math. Phys. 22, 89-103 (1971)

[Ge] Georgii, H.-O.: Private communication, unpublished

[GLM] Giacomin, G., Lebowitz, J.L., Maes, C.: Agreement Percolation and Phase Coexistence in Some Gibbs Systems. Preprint

[Gr] Grimmett, G.: Percolation, Heidelberg, New York: Springer, Berlin, 1989

[GS] Givens, J.A., Stell, G.: The Kirkwood-Salsburg Equation for Random Continuum Percolation. J. Stat. Phys. 59, 981-1018 (1980)

[H] Harris, T.E.: A Lower Bound for the Critical Probability in a Certain Percolation Process. Proc. Camb. Phil. Soc. 56, 13-20 (1960)

[Ho] Holley, R.: Remarks on the FKG Inequalities. Commun. Math. Phys. 36, 227-231 (1974)

[J] Janson, S.: Bounds on the Distribution of Extremal Values of a Scanning Process. Stoch. Proc. Appl. 318, 313-328 (1984)

[K] Klein, W.: Potts-Model Formulation of Continuum Percolation. Phys. Rev. B 26, 2677-2678 (1982)

[L] Liggett, T.M.: Interacting Particle Systems. Heidelberg, New York: Springer, Berlin, 1985

[LG] Lebowitz J.L., Gallavotti, G.: Phase Transitions in Binary Lattice Gases. J. Math. Phys. 12, 1229-1233 (1971)

[LM] Lebowitz, J.L., Monroe, J.L.: Inequalities for Higher Order Ising Spins and for Continuum Fluids. Commun. Math. Phys. 28, 301-311 (1972)

[LL] Lebowitz, J.L., Lieb E.H.: Phase Transitions in a Continuum Classical System with Finite Interactions. Phys. Lett. 39A, 98-100 (1972)

[P] Preston, C.: Spatial Birth-and-Death Processes. Bull. Inst. Inter. Stat. 46 (2), 371-391 (1975)

[R] Ruelle, D.: Existence of a Phase Transition in a Continuous Classical System. Phys. Rev. Lett. 27, 1040-1041 (1971)

[Ru] Russo, L.: An Approximate Zero-One Law. Zeitschrift für Wahrscheinlichkeitstheorie and Ihre Grenzgebiete 61, 129-139 (1982)

[WR] Widom, B., Rowlinson, J.S.: New Model for the Study of Liquid-Vapor Phase Transitions. J. Chem. Phys. 52, 1670-1684 (1970) 
